\title{
Study on Rapid Methods for Detecting Foodborne Pathogens in Frozen Meat
}

\author{
Junling Wang ${ }^{1}$, Chuang Yang ${ }^{1}$, Tiantian Wang ${ }^{1}$, Kunyu Liu ${ }^{1}$, Yawei Xu ${ }^{1}$, Rui Yin ${ }^{1}$, Huaizhi \\ Tang ${ }^{1}$ \\ ${ }^{1}$ Jilin Agricultural Science and Technology University, Jilin, Jilin Province, China
}

Keywords: foodborne pathogenic microorganisms; PCR; sensitivity; specificity

\begin{abstract}
In this paper, food borne pathogenic microorganisms are taken as the target bacteria. By using the PCR technique, a fast and efficient PCR reaction system is established to detect pathogenic microbes in frozen meat. The sensitivity and specificity of this reaction system are detected and analyzed; the most suitable method for rapid detection of pathogenic microorganisms in food is obtained.
\end{abstract}

\section{Introduction}

With the development of national economy, the scale of food industry is expanding in China. However, in recent years, food borne pathogens which can cause food borne diseases are common seen. [1] Among all these food borne pathogens, microorganisms lead to the greatest numbers of disease and patients, accounting for $40.93 \%$ and 56.39\% respectively. [2] China is a major producer and consumer of meat products. Meat products are susceptible to contamination caused by various microorganisms and can breed many kinds of pathogenic bacteria. [3] Escherichia coli are common food borne pathogen in meat; they can pollute all kinds of meat products. The Ministry of Science and Technology issued a guideline for key projects named Research and Development of Food Safety Technologies, and mentioned that from 2017 to 2021, 15 to 20 kinds of techniques should be developed to realize the rapid detection of pathogens and toxins in food; 15 to 20 new types of high throughput testing kit and 5 to 8 sets of equipments should be built to improve the technical and equipment levels of China in disease microbe detection. Obviously, rapid detection technique is the focus of foodborne pathogen detection technology.

For a long time, in laboratory, the detection of pathogen is realized through traditional bacteriological detection methods. The national standard measure of traditional separation method is still widely used in detection agencies. But that measure requires long time period and multiple links; it often takes several days to draw the conclusion. [4] Meanwhile, the sensitivity of this method is low. Miss and false detections usually happen. The traditional detection method cannot meet the requirements of rapid food detection in modern society. [1] Immunological detection can combine highly specific antigen-antibody reaction with highly sensitive enzyme catalysis; it also has shortcomings. The immunological detection method requires a suitable number of purified bacteria, or high concentration of bacteria obtained through concentrated samples. So a long preparation period is needed; the results cannot be got in short time. [5] In recent years, polymerase chain reaction (PCR) is the most widely used molecular biology technique. In this method, specific primers are amplified by highly conservative nucleic acid sequence; the results are observed through gel electrophoresis and ultraviolet nucleic acid detector. [6]

Based on PCR technology, this paper detected the contents of Escherichia coli contained in frozen meat bought from the market, trying to establish a set of rapid, efficient and accurate method to detect Escherichia coli in frozen meat, and provide reference for the rapid detection of foodborne pathogenic microorganisms. The main contents included three parts: the establishment of Escherichia coli PCR reaction system; the optimization of reaction conditions for the annealing temperature of Escherichia coli PCR; the detection of samples to determine the sensitivity of PCR method and the specific analysis of PCR. 


\section{Research Materials and Method}

\subsection{Research materials and equipment}

Research materials included Escherichia coli (isolated by the microbiological laboratory of Jilin Agricultural Science and Technology University); Taq enzyme and DNA Marker DL5000 (purchased from BioTeke Biotechnology Co., Ltd.); frozen meat; SW-079PCR analyzer (produced by TaKaRa Biologic Engineering Co., Ltd. in Dalian); JY600t electrophoresis apparatus (produced by Beijing Jun Yi Oriental Electrophoresis Co., Ltd.); LAS 500 biology molecular imager (produced by Beijing Lumiere Science and Technology Co., Ltd.); FA1004A electronic scale (produced by Shanghai Jing Tian Electronic Instrument Co., Ltd.); SW-073 two person single side purification work bench (produced by Suzhou Purification Equipment Co., Ltd.); HH-8 digital display constant temperature water bath (produced by Jintan Science and Analysis Instrument Co., Ltd.); HZQ-X10CA constant temperature oscillating incubator (produced by Shanghai Yiheng Technology Co., Ltd.); FMB20 ice maker (produced by Shanghai Bilang Co., Ltd.); 5424R (frozen) Eppendorf desktop high-speed centrifuge (produced by Shanghai Queensland Biotechnology Development Co., Ltd.) and PHB-10 acidity meter (produced by Shanghai Danding International Trade Co., Ltd.). Other chemical reagents were chemically pure.

\subsection{Research Method}

Design of primers. Escherichia coli encoded hemolysin gene (hlyA) was selected as the target gene. Primer 5.0 analysis software was used to design primers respectively. The upstream primers were $\mathrm{F}$ 5'-GCATCATCAAGCGTACGTTCC-3` and the downstream primers were $\mathrm{R}$ 5`AATGAGCCAAGCTGGTTAAGC-3`. The primers were synthesized by Jinruisi Bioscience Limited Company.

Culture of strain. The preserved strains were taken out and quickly defrosted in water at 37 degrees; $100 \mu \mathrm{l}$ was inoculated to $100 \mathrm{ml} \mathrm{LB}$ liquid medium. At 37 degrees centigrade, the strains were cultivated through shake cultivation method over night. The culture medium was streak inoculated on LB solid medium; single colonies were isolated. Single colonies on the plates were inoculated on LB liquid medium and cultured to logarithmic growth phase for following experiments.

Extraction of genome DNA. The improved CTAB method created by Xin-mei Guo, Ji-chuan Kang and their colleagues was adopted in this experiment. [7] $1 \mathrm{~mL}$ logarithmic period bacteria liquid was extracted and centrifuged for 5 minutes at $12000 \mathrm{r} / \mathrm{min}$. $30 \mu \mathrm{L} 10 \%$ SDS was added to the bacterial precipitation, followed by $150 \mu \mathrm{L}$ CTAB extraction buffer (Tris- $\mathrm{HCl} 50 \mathrm{mmol} / \mathrm{L}$, $\mathrm{pH}$ value 8, EDTA $10 \mathrm{mmol} / \mathrm{L}$; $\mathrm{NaCl} 0.7 \mathrm{~mol} / \mathrm{L}$; CTAB 1.5\%). $5 \mu \mathrm{L}$ chloroform $\mathrm{K}$ and CTAB/NaCl $80 \mu \mathrm{L}$ were added for water bath at 60 degree for 40 minutes. The isometric phenol: chloroform: isoamyl alcohol (25: 24: 1) were added and gently mixed, then centrifuged for 5 minutes at the speed of 12000 $\mathrm{r} / \mathrm{min}$ at 4 centigrade. The supernatant was taken and mixed with $400 \mu \mathrm{L}$ isopropanol, then centrifuged for 5 minutes at $12000 \mathrm{r} / \mathrm{min}$ at 4 centigrade. Then the supernatant was abandoned, adding $70 \%$ ethanol to wash and precipitate. The precipitation dried naturally. $100 \mu \mathrm{L}$ TE with RNA enzyme A (20 mg/mL) was used to dissolve DNA. The material was frozen at minus 20 degrees for storage.

Pretreatment of food samples and extraction of template DNA. Sample processing: under sterile conditions, $25 \mathrm{~g}$ or $25 \mathrm{~mL}$ food samples were taken for homogeneity in $225 \mathrm{~mL}$ sterilized normal saline. 10 times gradient diluents were obtained.

Enriched culture: the bacteria solution was seeded in $7.5 \% \mathrm{NaCl}$ broth or peptone broth, and then oscillated for half an hour at 37 degrees.

At the speed of $4000 \mathrm{r} / \mathrm{min}$, the bacterium was centrifuged for 10 minutes at 4 degrees. The supernatant was introduced to another sterilization centrifuge tube and centrifuged with $10000 \mathrm{rpm}$ for 20 minutes. The precipitation was extracted according to the bacterial genome DNA extraction procedure; the established PCR reaction program was used to detect it. The electrophoresis of PCR products on agarose gel was achieved and then observed by the gel imaging system.

Analysis of sensitivity and specificity. After the enriched culture, pathogenic microorganisms 
were inoculated into food samples and counted. The bacteria solutions were diluted with physiological saline to get diluted solutions at 10-1, 10-2, 10-3, 10-4, 10-5, 10-6 and 10-7 times. $2 \mathrm{~mL}$ diluents were used to extract DNA. The extracted DNA was used as the template for PCR detection.

\section{Research Results and Analysis}

\subsection{Optimization of PCR reaction conditions}

The optimization of PCR annealing temperature: in order to select the best annealing temperature, the researcher referred to previous studies and selected the temperature points of 47.3 degrees, 48.4 degrees, 50.0 degrees, 51.7 degrees, 53.3 degrees and 54 degrees for multiple PCR amplification. The results were shown in Figure 1 . With the increasing of annealing temperature, the strips became obviously brighter. The increasing trend stopped at 53.3 degrees. Therefore, the optimum annealing temperature for multiple PCR was determined as 53.3 degrees.

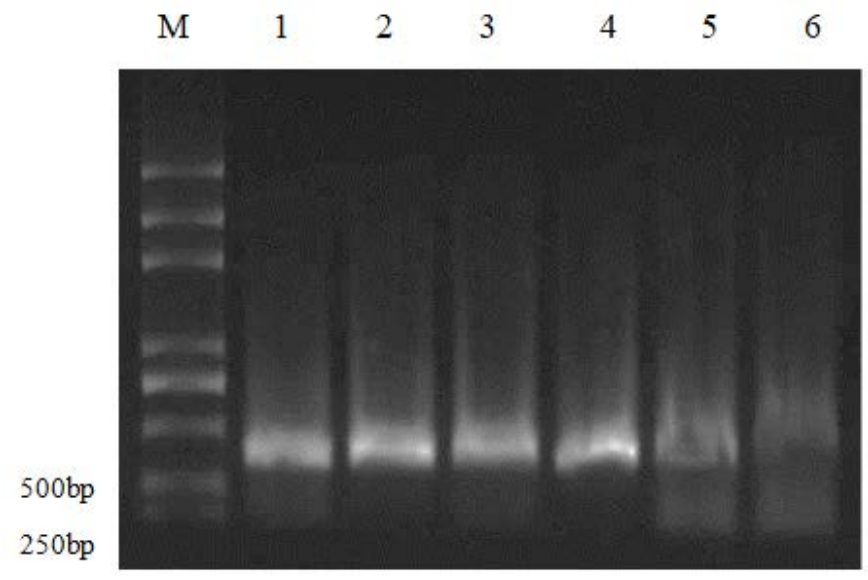

Figure 1 Results of PCR products detection under different annealing temperatures

M: DNA Marker 5000bp Ladder; $1.47 .3^{\circ} \mathrm{C} ; 2.48 .4^{\circ} \mathrm{C} ; 3.48 .4^{\circ} \mathrm{C} ; 4.50 .0^{\circ} \mathrm{C} ; 5.51 .7^{\circ} \mathrm{C} ; 6.53 .3^{\circ} \mathrm{C} ; 7.54 .0$

\subsection{Detection of artificial polluted samples}

The food was processed according to method described in the 2.2.4 part of this paper; the DNA of samples was extracted. The DNA extracted from the frozen meat was amplified by PCR. The results of observation were shown in Figure 2. The specific bands of amplified strains of required bacteria were clear and bright, indicating the existence of Escherichia coli in food and the successful result of amplification.

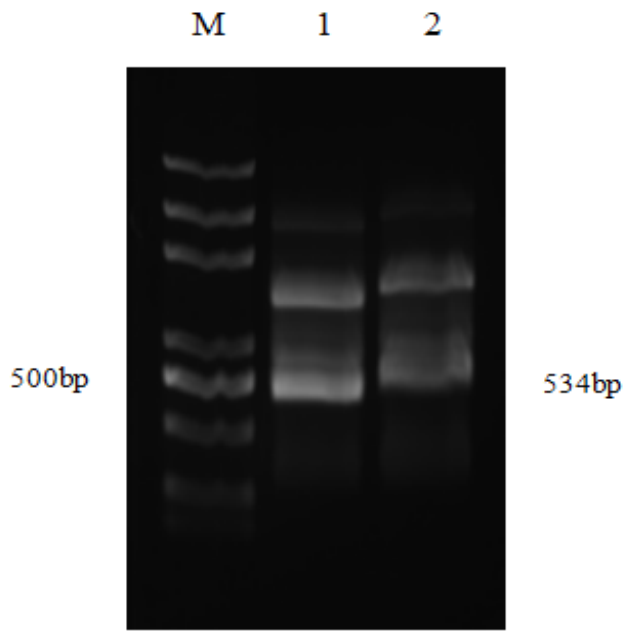

Figure 2. PCR detection results of frozen meat inoculated with Escherichia coli

M: DNA Marker 5000bp Ladder; 1 2: Escherichia coli 


\subsection{Sensitivity inspection of PCR sample}

The conditioning fluid of frozen meat which was inoculated with escherichia coli was cultured and used to extract DNA. Using DNA as the template, 10 times gradient dilution and PCR detection were carried out. From Figure 3, the minimum detection limit of Escherichia coli was $2 \times 10^{-4} \mathrm{ng} / \mu \mathrm{L}$.

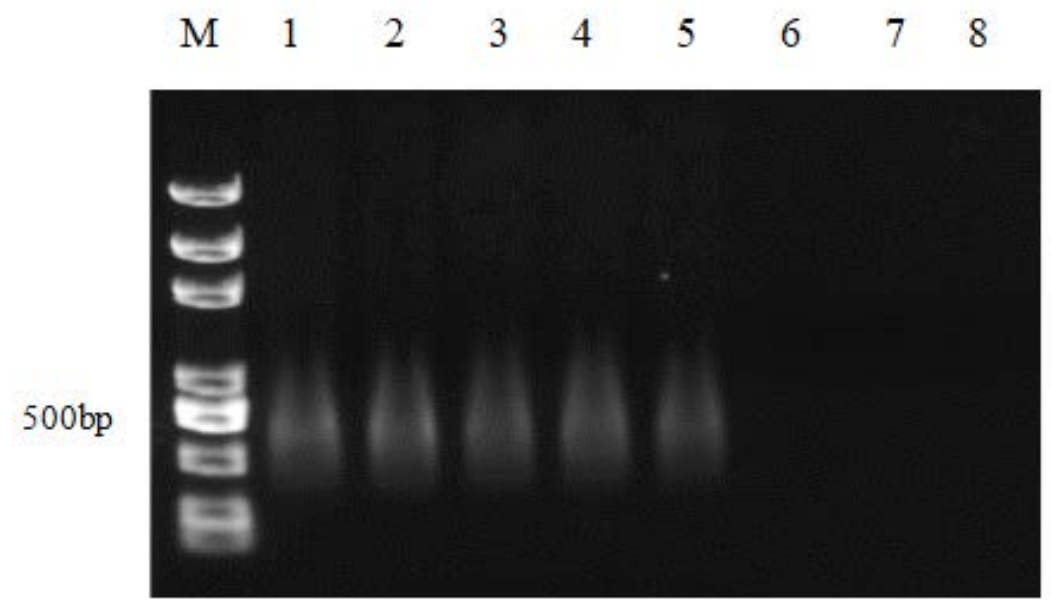

Figure 3. Results of PCR sensitivity test for Escherichia coli in samples

M: DNA Marker 2000bp Ladder; $1.10 ; 2.10^{-1} ; 3.10^{-2} ; 4.10^{-3} 5.10^{-4} ; 6.10^{-5} ; 7.10^{-6} ; 8.10^{-7}$.

\section{Conclusion and Discussion}

According to the results of this experiment, the selected hemolysin gene (hlyA) of Escherichia coli can be used as the specific primer of target gene design. The specificity of primers is strong and does not interfere with each other. It can be used in the detection of Escherichia coli through PCR. When optimize the annealing temperature of PCR reaction system, it was found that the optimum annealing temperature was 53.3 degrees. The lowest detection content of E. coli in frozen meat samples was $2 \times 10^{-4} \mathrm{ng} / \mu \mathrm{L}$. In this study, Escherichia coli had strong specificity in frozen meat.

In this study, the design of specific primer was not flawless. There was an unpredictable strip in the control group of PCR Escherichia coli, though it did not affect the identification of Escherichia coli. The selection of specific target gene was particularly important. In this experiment, the minimum detection limit of Escherichia coli sensitivity was low. Two influencing factors were found after discussion and analysis. The concentration and content of genomic DNA in samples were low; the contents of fat and protein in frozen meat were high. The medium composition affected the PCR results. However, the infective doses of most meat products were more than 103CFU/ml. [8] The sensitivity test results of this experiment have reference significance and practical application prospects.

This experiment established a PCR method to detect Escherichia coli in frozen meat, which greatly shortened the inspection time and lowered the cost. The detection method has characteristics of high specificity, high sensitivity and low cost. Compared with the traditional detection method used in laboratory, it saves a lot of time and has certain practical values in food safety detection.

\section{Acknowledgement}

Fund Project: This paper is supported by the Foundation for Initial Funding Projects for Doctors of Jilin Agricultural Science and Technology University (No. B01 Document of Jilin Agricultural Science and Technology University published in 2016). It is also supported by the Foundation for Undergraduates' Innovative Research Projects of Jilin Agricultural Science and Technology University (No. 020 Document of Jilin Agricultural Science and Technology University published in 2017). 


\section{References}

[1] X.Y. Liu, Y.X. Hu, Current situation of foodborne diseases in the world, J. Foreign Medical Sciences (Section of Hygiene). 30 (2003).

[2] J.F. Xu, J.Z. Zhang, Analysis of foodborne diseases outbreak in China from 2001 to 2010, J. Chinese Agricultural Science Bulletin. 28 (2012).

[3] M.Y. Li, G.H. Zhou, X.L. Xu, et al. Changes of bacterial diversity and main flora in chilled pork during storage using PCR-DGGE, J. Food Microbiology. 23 (2006).

[4] W.J. Wang, F.D. Meng, Development of fast detection kit for foodborne pathogenic bacteria, Fourth Symposium on Swine Disease Prevention and Control organized by the Animal Infectious Diseases Branch of Chinese Association of Animal Science and Veterinary Medicine, 2010.

[5] H.Y. Sun, Establishment of a Multiplex PCR Detection Method for Four Foodborne Pathogens, School of Public Health, Jilin University, 2011.

[6] X.Y. Cheng, The Development of Rapid Detection Technique for Several Foodborne Pathogens, Food Science and Engineering, Ocean University of China, 2012.

[7] X.M. Guo, J.C. Kang, J. Zhang, DNA Extraction of Staphylococcus aureus using the CTAB method, J. Journal of Mountain Agriculture and Biology. 24 (2005).

[8] R.Y.C. Kong, S.K.Y. Lee, T.W.F. Law, et al. Rapid detected of six types of bacterial pathogens in marine waters by multiplex PCR, J. Water Research. 36 (2002). 